Lepr Rev (1992) 63, Supplement, 99s-105s

\title{
The relevance of future leprosy vaccines to disease control
}

\author{
M D GUPTE
}

The concepts on leprosy control vary widely, according to different perceptions of the problem, the technology available to meet the targets and the commitment to achieve the final goal. The Executive Board of the World Health Organization, in its 82nd session in January 1991, and the World Health Assembly in May 1991 passed resolutions to eliminate leprosy, i.e., to achieve a prevalence of less than 1 per 10,000, by the year 2000 . Leprosy is a public health problem because of the associated deformities and the resultant negative social overtones. Worldwide, leprosy control programmes were aimed at the early detection and treatment of leprosy, to enable the prevention of disabilities. As a secondary effect of chemotherapy, it was hoped that the transmission of leprosy infection would be lowered, resulting in better control of the disease. Multidrug therapy, as a newer strategy, essentially focuses on the same theme.

In the 1950s dapsone was the first drug to be used in public health practice for leprosy control. With dapsone, a comparatively rapid decline in leprosy prevalence was observed in several sets of population-based data. It was unclear whether the recorded decline and eventual incidence stabilization was because of backlog clearance or was the effect of dapsone. ${ }^{1}$ Dapsone was also tested as a chemoprophylactic agent in several places-in this role it could be considered only in individual situations, and not as a general public health measure for mass prophylaxis. ${ }^{2}$ The probable role of dapsone in neuropathy ${ }^{3}$ and the widespread problem of dapsone resistance ${ }^{4}$ further emphasized the limitations of the application of dapsone therapy in respect to leprosy control.

BCG was considered as a potential tool for leprosy control following the observations of Fernandez that demonstrated lepromin conversion. ${ }^{5}$ Encouraging results of its protective efficacy are available from Uganda, New Guinea and Malawi - a prophylactic efficacy of $50-80 \%$ was observed there, ${ }^{6}$ - but results from Burma have shown an efficacy of about $20 \% .^{7}$ A similar moderate level of protective efficacy was observed from the recently analysed data from the South Indian BCG trial. ${ }^{8}$ In this trial, a $0 \cdot 1 \mathrm{mg}$ dose of BCG consistently gave higher protection than a $0.01 \mathrm{mg}$ dose, the overall efficacy being $24 \%$ and $17 \%$, respectively, for these two doses. It was also seen that protective efficacy was highest in the youngest age group, indicating the probable efficacy of BCG in the uninfected population. Both sexes had the same protection. Protective efficacy of BCG did not differ greatly in PPD-S and PPD-B positive and negative groups. Protective efficacy against various clinical forms of leprosy was similar, except in the pure neuritic type, where it was somewhat lower. The smear positive incidence cases detected in the trial were uniformly distributed among those receiving doses of $0.1 \mathrm{mg} \mathrm{BCG}, 0.01 \mathrm{mg} \mathrm{BCG}$ or 
a placebo. This indicates that smear positive leprosy cases were not prevented by BCG. Thus BCG does not appear to be a vaccine which could be used for the general prevention of leprosy, although it might prevent leprosy in some parts of the world.

Multidrug therapy campaigns have recorded remarkable success in terms of prevalence reduction in several parts of the world. ${ }^{9}$ We examined district level data in India, where MDT campaigns were operative for over 8 years, but there was no apparent indication that a declining incidence could be attributed to MDT. If leprosy is eventually controlled or 'eliminated' by the use of MDT, it will be the second disease to be overcome by chemotherapy, the first being yaws.

It seems obvious, considering these facts, that an effective vaccine against leprosy is needed. At the moment there are three or four antileprosy vaccine candidates being tested, and field trials with these vaccines are underway. The advances in molecular biology have made it possible for there to be second generation vaccines. Hence, when vaccines for preventing leprosy are considered, ICRC, Mycobacterium welchii, M. leprae combined with BCG and possible second generation vaccines are favourites as the candidates. One more vaccine, M. habana, is in the process of development, but there is not enough information available yet on its possible role as an immunoprophylactic agent against leprosy.

\section{LEPRAE (A) IN COMBINATION WITH BCG}

Kirchheimer \& Storrs ${ }^{10}$ in the early 1970s reported a major advance in experimental leprosy - the nine-banded armadillo, Dasypus novemcinctus, model for the cultivation of $M$. leprae. Following this large quantities of $M$. leprae have become available, and scientists have begun to develop $M$. leprae-based vaccines. A safe supply of $M$. leprae for immunological studies in man was obtained. ${ }^{11}$ There is no single batch for M. leprae preparations, they are all harvests and lots. ${ }^{12}$ Lots tested in various vaccine trials in the world have demonstrated the capacity of the vaccine to produce sensitization against $M$. leprae. Various lots of $M$. leprae vaccine have also demonstrated protection in the mouse footpad model. Characterization of different harvests are difficult.

Since 1989 , we have used varying doses of BCG $+M$. leprae in vaccine studies in Thiruthani Taluk, Chingleput district, Tamilnadu. In several other studies on man in different parts of the world, a $M$. leprae + BCG combination has also been observed to have sensitizing potential in terms of lepromin and soluble antigen skin test conversions.

Sensitizing potential and protection against footpad infection in mice following inoculation with $M$. leprae was demonstrated by Shepard. ${ }^{13}$ Studies in the Central JALMA Institute for Leprosy (CJIL), Agra, demonstrated that the combination of BCG and $M$. leprae was effective in preventing $M$. leprae infection in the mouse model. ${ }^{14}$

Convit ${ }^{15}$ demonstrated the immunotherapeutic effect of combined killed $M$. leprae and BCG in a study involving 155 inactive BL/LL patients, 351 active BL/LL patients, and 46 indeterminate leprosy patients, findings that were independently confirmed by a group of histopathologists.

\section{W. VACCINE}

Talwar's group in Delhi looked for a mycobacterium that not only contained desirable cross-reactive antigens with $M$. leprae with respect to the immune reactivity of TT 
patients, but also contained antigens evoking response in LL patients. Based on their studies. M.w. was selected as a candidate for vaccine production. ${ }^{16}$

\section{Safety}

Talwar carried out animal toxicity studies for M.w. vaccine and the product was found safe. M.w. has been used in several parts of India on patients, and is also being tested in a field study at Kanpur. M.w. vaccine has been well tolerated by the vaccinees and there are no reports of side- or toxic effects. Being a cultivable organism, it is possible to identify various characteristics of the bacillus and quality control is easy.

\section{Animal studies}

The CJIL studies demonstrated a heightened DTH response and strong protection in the mouse model. ${ }^{14}$

\section{Sensitization studies}

Talwar observed the conversion rate following M.w. vaccination was $90-98 \%$ in the lepromin negative contacts of LL patients. ${ }^{17}$ In a study we carried out, M.w. vaccine was used in a dose of $10^{9}$ bacilli and $5 \times 10^{9}$ bacilli. We found that with the increased dose of $5 \times 10^{9} M . w$, post-vaccine responses to Rees' skin test antigen and lepromin-A were marginally higher than the responses observed in the normal saline group.

\section{Immunotherapy}

Phase-I clinical trials revealed that 20 of $32 \mathrm{BL} / \mathrm{LL}$ patients, persistently negative to Dharmendra and Mitsuda lepromins, were converted to a lepromin positivity status with a single intradermal injection of $5 \times 10^{7}$ autoclaved M.w. This was still manif est $8-11$ months after the initial immunization. ${ }^{18}$ Hospital-based Phase-II immunotherapeutic clinical trials have been in progress since December 1986 in two hospitals in New Delhi. Talwar observed success, based on results on 103 patients belonging to the 'single blind' portion of this study. ${ }^{19}$

\section{ICRC VACCINE}

ICRC bacilli were first isolated in 1958 by Bapat et al. ${ }^{20}$ ICRC vaccine was first produced in 1979, and Bapat and Deo registered a patent for ICRC vaccine (C-44 strain) in 1981. The initial hospital-based studies were conducted from 1979 at the Acworth Leprosy Hospital, Bombay. A prophylaxis study has been in progress in Maharashtra State since February $1987 .^{11}$

\section{Safety}

Extensive animal toxicology studies were conducted on the ICRC vaccine at the Cancer Research Institute, Bombay. ICRC vaccine has been used on leprosy patients since 1979. In the immunoprophylaxis trial in Western Maharashtra, India (30,000 population), 
leprosy patients developed no complications following ICRC vaccine, apart from an extremely small number of individuals who developed regional suppurative adenitis. ICRC vaccine, like $M . w$, is a product developed from cultivable bacilli. The quality of this product should be maintained easily.

\section{Animal studies}

Bhide found that ICRC vaccine provided protection against $M$. leprae infection in the mouse model, ${ }^{22}$ but these results were unconfirmed by Sreevatsa \& Desikan. ${ }^{14}$

\section{Sensitization}

A total of $11(92 \%)$ out of 12 lepromin negative individuals were converted following ICRC vaccine. ${ }^{23}$

\section{Immunotherapy}

In 1981, Deo and colleagues published their findings on the potential antileprosy vaccine from killed ICRC bacilli. ${ }^{24}$ The 'vaccine' was administered to $46 \mathrm{LL}$ and $11 \mathrm{BB} / \mathrm{BL}$ patients who were on dapsone treatment, and 10 lepromatous patients with high BI also received rifampicin for varying periods. Similarly treated 9 LL patients, who received saline, served as controls. A total of 14 of $46 \mathrm{LL}$ vaccinated patients developed ENL. Histopathological examination of skin biopsies from vaccinated LL patients revealed regressive changes, and 4 months after vaccination, lepromin conversion was observed in $50 \%$ and $80 \%$ of $\mathrm{LL}$ and $\mathrm{BB} / \mathrm{BL}$ cases, respectively. Bhatki et al. ${ }^{25}$ reported 5 cases of reversal reactions induced by ICRC vaccine in LL patients, providing additional evidence of the immunotherapeutic potential of ICRC vaccine. A group of LL patients is being investigated for T-cell responses of ICRC and $M$. leprae antigens before and 6-10 months after vaccination with ICRC vaccine. It was observed that in 12 out of 16 vaccinated patients, there was a drop in the bacillary index after vaccination- 6 of them showed parallel increase in the proliferative responses to both ICRC and $M$. leprae antigens. ${ }^{26}$

\section{Field trials}

Field trials using ICRC, M.w. and M. leprae+BCG vaccines have been launched worldwide. M. leprae + BCG is being tried in Venezuela and Malawi. ICRC and M.w. vaccines are being tried in Maharashtra and Uttar Pradesh respectively for their prophylactic efficacy. In January 1991, a comparative vaccine trial involving all these vaccines was launched by the Indian Council of Medical Research in the ChengalpattuMGR district of Tamilnadu. The first results from Venezuela are expected in the near future. Results from the other trials can be expected after 1994. Vaccine trials are very demanding exercises and results from field trials will be awaited with great interest.

\section{Possible second generation vaccines}

A number of mycobacterial antigens $(70 \mathrm{KD}, 65 \mathrm{KD}, 31 \mathrm{KD}, 18 \mathrm{KD}$ and $10 \mathrm{KD})$ have been 
identified. Natural or recombinant forms of these proteins are now available. Choosing antigens with possible prophylactic efficacy could prove to be a very deceptive exercise. Activity is focused on secretory proteins which are also the most prominent proteins in bacterial extracts. Defining 'protective antigens', and 'protective and pathologic immunity' are some of the questions that are being investigated (D B Young, 'Defining protective antigens', working paper for Joint PVD/TDR Meeting on Mycobacteria, Geneva, 1 May 1991). Promising approaches for inserting different DNA sequences in BCG have been developed. ${ }^{27}$ As with other second generation vaccines, other carriers such as Canary-pox virus, salmonella, attenuated listeria, etc., could also be considered. The use of alum and other adjuvants is generally the initial approach used. The work on second generation vaccines is still very much at the exploratory stage. The studies based on cytokine liberation may be employed to identify some of the potential antigens in a comparatively short period of 1 or 2 years. However, it is difficult to postulate the availability of second generation vaccines and their potential role in the near future, even for field trials.

\section{Effects of therapy and vaccine}

The direct or primary effect of chemotherapy in leprosy is antimicrobial. The bacteria are killed rapidly, which leads to a cure. As the bacteria in patients are killed, the load of infection is reduced. This effect is expected to lead to the reduction in the risk of infection and also to lessen risk of disease-both secondary effects of chemotherapy. However, in view of the long latent/incubation period, new cases will keep occurring for some years. Therefore some time will elapse before the full impact of chemotherapy on the incidence of leprosy will be established. But as can be seen from the available data, no perceptible impact has been noticed even after 7 or 8 years of MDT. Thus the secondary effect of therapy on reducing transmission of leprosy cannot be presumed, though it is theoretically possible.

Therefore, prophylactic vaccines have a place in controlling leprosy transmission. BCG acts against leprosy by preventing primary infection and also reinfection. BCG may not be effective against reactivation. The candidate vaccines presently available are supposed to have immunotherapeutic efficacy. Hence they might be effective in infected individuals and they may also prevent the reactivation type of disease. A vaccine that is effective in both uninfected and infected individuals is likely to have the direct and rapid effect of reducing the incidence of leprosy in an area with active $M$. leprae transmission. In an area with a low level of transmission, vaccination will be a potent tool in reducing the number of those susceptible.

\section{Relevance of a vaccine}

The available parameters of animal studies, sensitization to $M$. leprae antigens following vaccination and immunotherapy, are only indirect measures of the probable prophylactic efficacy of the vaccines that are being developed. There are now vaccine trials testing each different vaccine. Several recombinant and native antigens as well as mycobacterial components are being investigated for their role in immuno-modulation. It is not 
impossible to conceive the emergence of effective antileprosy vaccines one day. Perhaps a single antileprosy vaccine may not be effective in all the geographical areas or in different epidemiological situations. It would be essential to understand the possible roles of these vaccines in different situations, if they are found to be effective prophylactic agents. Whether the measure could be applied generally to the total population, or only to certain high risk groups needs to be ascertained. The assessment of the risk of disease and the risk of complications following vaccination is needed. The possible benefits of prevention will have to be considered. A vaccine may prevent only the early and less infectious forms of leprosy. It may be necessary to have a vaccine that will also prevent serious and highly infectious forms. Alternative approaches and priorities for disease control will have to be taken into account. Case detection and case holding in controlling disease transmission, costs of case detection and case holding and the cost of preventing leprosy cases will need consideration in an overall context. Clearly leprosy vaccine is a distinct research goal and an area of high research priority.

\section{References}

1 WHO Study Group. Epidemiology of leprosy in relation to control. Technical Report Series 716. WHO: Geneva, 1985, 35-6.

2 Noordeen SK. Chemoprophylaxis against leprosy. In: A window on leprosy, Chatterjee BR (ed). Delhi: Gandhi Memorial Leprosy Foundation Silver Jubilee Commemorative Volume, 1978; 355-60.

${ }^{3}$ Gupte MD. Dapsone treatment and deformities: A retrospective study. Lepr Ind, 1979; 51: 218-35.

4 WHO Study Group. Chemotherapy of leprosy for control programmes. Technical Report Series 675. WHO: Geneva, 1982, 9-14.

5 Fernandez JMM. Estudio comparative de la reaction de Mitsuda con las reacciones tuberculincas. Rev Argent Dermatosif, 1939; 23: 425-53.

6 Bagshawe A, Scott GC, Russell DA, Wigley SC, Merianos A, Berry G. BCG vaccination in leprosy: final results of the trial in Karimui. Papua New Guinea, 1963-79. Bull Wld Hlth Org, 1989; 67: 389-99.

${ }^{7}$ Kyaw Lwin, Sundaresan T, Mg Mg Gyi, Bechelli LM, Tamondong C, Gallego Garba josa P, Sansarricq H, Noordeen SK. BCG vaccination of children against leprosy: fourteen year findings of the trial in Burma. Bull Wld Hlth Org, 1985; 63: 1069-78.

8 WHO. Report of the Joint IMMLEP/THELEP meeting on Field Research in leprosy, Geneva, September 1990; 1-17.

9 Noordeen SK, Lopez Bravo L, Daumerie D. Global review of multidrug therapy (MDT) in leprosy. Wrld Hlth Stat, 1991; 44: 2-15.

10 Kirchheimer WF, Storrs EE. Attempts to establish the armadillo (Dasypus novemcinctus, Linn.) as a model for the study of Leprosy. Report of lepromatoid leprosy in an experimentally infected armadillo. Int J Lepr, 1971; 39: 693-702.

11 Rees RJW. Progress in the preparation of anti-leprosy vaccine from armadillo-derived Mycobacterium leprae. Int J Lepr, 1983; 51: 515-18.

12 World Health Organization (1981). Testing of purified armadillo-derived M. leprae in man (Document finalized by the IMMLEP Steering Committee at its meeting, 10-12 June 1981). TDR/IMMLEP/SC/TEST/ 81.1 .

13 Shepard CC. Animal vaccination studies with Mycobacterium leprae. Int J Lepr, 1983; 51: 519-23.

14 Sreevatsa, Desikan K V. Evaluation of the efficacy of candidate vaccines against $M$. leprae infection in mice. Ind J Lepr, 1988; 60: 252-9.

15 Convit J, Aranzazu N, Ulrich M, Zuniga M, De Aragon ME, Alvarado J, Reyes O. Investigations related to the development of a leprosy vaccine. Int J Lepr, 1983; 51: 531-9.

16 Talwar GP, Fotedar A. Two candidate anti-leprosy vaccines: current status of their development. Int J Lepr, 1983; 51: 550-2.

17 Talwar GP. Vaccine based on M.w. bacillus. Paper presented in Pre-congress Workshop on Leprosy vaccine trials, The Hague, The Netherlands, 1988.

18 Chaudhary S, Fotedar A, Talwar GP. Lepromin conversion in repeatedly lepromin negative BL/LL patients af ter immunization with autoclaved Mycobacterium w. Int J Lepr, 1983; 51: 159-68.

19 Talwar GP, Zaheer SA, Suresh NR, Parida SK, Mukherjee R, Singh IG, Sharma AK, Kar HK, Misra RS, 
Mukherjee A. Immunotherapeutic trials with a candidate anti-leprosy vaccine based on Mycobacterium $w$. Indo-EC Symposium on Leprosy \& Mycobacterial Disease, 1989; 39-41.

20 Bapat CV, Ranadive KJ, Khanolkar VR. In vitro cultivation of an acid fast mycobacterium isolated from human lepromatous leprosy. Ind J Pathol Bacteriol, 1958; 1: 156-9.

${ }^{21}$ Kartikeyan S, Chaturvedi RM, Deo MG. The sociocultural dimension in leprosy vaccine trials. Lepr Rev, 1990; 61: 50-9.

22 Bhide MB, Pradhan KS, Bapat CV. A vaccine from ICRC bacilli: against $M$. leprae injection in mouse footpad. Lepr Ind, 1978; 50: 334-44.

23 Deo MG, Bapat CV, Bhalerao V, Chaturvedi RM, Bhatki W, Chulawala RG. Anti-leprosy potentials of ICRC vaccine. A study in patients and healthy volunteers. Int $J$ Lepr, 1983; 51: 540-9.

24 Deo MG, Bapat CV, Bhatki WS, Chulawala RG. Potential anti-leprosy vaccine from killed ICRC bacilli-a clinicopathological study. Ind J Med Res, 1981; 74: 164-77.

25 Bhatki WS, Chulawala RG, Bapat CV, Deo MG. Reversal reaction in lepromatous leprosy patients induced by a vaccine containing killed ICRC bacilli: A report of five cases. Int J Lepr, 1983; 51: 466-72.

26 Gangal SG, Chiplunkar SV, Shinde SR, Samson PD, Deo MG. Immunoreactivity of T-cells from leprosy patients to ICRC and M. leprae antigens before and af ter vaccination. Trop Med Parasit, 1990; 41: suppl 2, $472-4$.

27 Bloom BR, Jacobs WR Jr. New strategies for leprosy and tuberculosis and for development of Bacillus Calmette-Guerin into a multivaccine vehicle. Biomedical Science and the Third World: Under the Volcano. Ann NY Acad Sci, USA, 1989; 569: 155-73. 